\title{
用成键轨道近似研究 $\mathrm{GaAs}(110)$ 表面声子
}

\author{
林子散 顾一鸣正克林
}

（中国科学技末大学基础物理中心, 合肥）

\section{一、引}

i：半导体表面原子并不处于由体内结构所决定的“理想”位置，而是出现各式各样的跑豫与 重构. 由于表面结构问题的复杂性，一个表面的确定往往需要各种方法相互印证. 尽管国际 上在这方面做了大量工作，但被公认已被解决的却极少。由于表面振动能提供表面几何结构 与表面电子结构相互关联的直接实验验证途经, 表面振动可能会在表面结构问题上起重要的 作用. 同时,表面振动对表面动力学行为及热力学性质均有重要影响,对其进行逐步深化的研 究是很需要的.

要了解表面振动,首先要求得晶体的动力学矩阵,这牵迩到体系总能对格点位移的二阶偏 微商. 由于从头计算还很不成熟,将原势法用于表面也有一些困难. 因此,目前尚无合适的方 阹求得. 人们通常用体的力常数代替表面力常数来预言表面声子, 或用力常数参数拟合表面 与体声子谱来观察力常数由体内到表面的变化.

鉴于参数化的紧束缚方法在求能带问题上很成功, Sokel ${ }^{[1]}$ 的工作则表明成键轨道近似 (忽略占据态与非占据态之间的耦合对能带能量的影响, 用于体内振动问题能得到比较满意的 结果). 因此我们认为可以用成键轨道近似来讨论表面振动问题, 以求在物理上对表面动力学 性质有一定了解.

第二节里闸明本文计算 GaAs（110）表面的基本方法,详细的数值计算结果列于第三节 中. 除给出清楚的表面声子模的图形外着重讨论了所得结果中的几个有意义之处.

最后在第四节结束语中简略谈了本文所用方法的优点、不足之处及改进的可能.

\section{二、理论方法简述}

$\mathrm{Chadi}^{[2]}$ 用参数化的紧束缚方法给出的一些半导体表面的重构得到了广泛的承认,其方法 也被用于一些晶体动力学问题,得出了较好的结果 ${ }^{[3]}$. 因此, 我们采用相似的方法,将体系总能 写为两部分:

$$
E_{t o t}-E_{b s}+U,
$$

这里 $E_{b s}$ 是电子能带结构能量, $U$ 为短程作用能并可用键长的相对变化展开 ${ }^{20}$, 即

$$
U-U_{c}+\sum_{i>i}\left(U_{1} \epsilon_{i j}+U_{2} \epsilon_{i i}^{2}\right)+\text { 高阶项, }
$$

式中 $i, i$ 是格点的标号, $\epsilon_{i j}-\frac{d_{i j}-d_{0}}{d_{0}}$ 是键长的相对变化昷, 其中 $d_{0}$ 是平衡键长, $d_{i j}$ 是 $i$ 
和 $i$ 格点振动时的相对键长. 求振动问题关键在于求出力常数矩阵

$$
\phi_{s a, v \beta}-\frac{\partial^{2} E_{t o s}}{\partial u_{s \alpha} \partial u_{v \beta}}-\frac{\partial^{2} E_{b s}}{\partial u_{s a} \partial u_{v \beta}}+\frac{\partial^{2} U}{\partial u_{s a} \partial u_{\nu \beta}},
$$

这里 $u_{\mathrm{Aa}}$ 指原胞中第 $\mathrm{k}$ 个原子的 $\propto$ 方向的位移. 要求出第二项是简单直接的, 但第一项的计算 却较困难, 因为格点位移导致布里洲区缺乏对称性,能带总能计算量显著增加, 求其二价偏饮 商就比较困难。但若采用成键轨道近似 ${ }^{(4)}$, 则:

$$
E_{b s}-\sum_{n k} E_{n}(k)-\sum_{i} E_{i}^{a \infty x},
$$

这里, $E_{i}^{o \infty}$ 是被占据的键轨道的能量, 求和是对所有的占据态进行的, 利用紧束线参数对键长 的平方反比依赖性 ${ }^{(4)}$, 很容易计算 $E_{i}^{o c c}$, 故 $E_{b s}$ 对动力学矩阵贡献很易求得.

在 $\mathrm{GaAs}_{\mathrm{a}}$ 体内,一元胞含两个原子即四个成键轨道, 每成键轨道的能量为

$$
E_{b s}=2 E_{b}=\left(\varepsilon_{h}^{G_{2}}+8_{h}^{A^{2}}\right)-2 \sqrt{\left(\frac{\varepsilon_{h}^{G 2}-8_{h}^{A^{2}}}{2}\right)^{2}+V_{2}^{A^{2}}},
$$

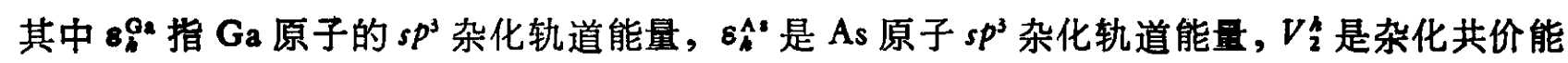
量, 由文献[4]可知 $V_{2}^{k}-\frac{33.3}{d^{2}} \mathrm{eV} \cdot \AA^{2}$. 由(5)式便可得到每个键轨道总能量

$$
E^{t o t}=U_{0}+U_{1} \epsilon+U_{2} \epsilon^{2}+E_{b s} \text {, }
$$

根据

$$
\left.\frac{\partial E^{t o t}}{\partial d}\right|_{d=d_{0}}=0,\left.\frac{\partial^{2} E^{t o t}}{\partial d^{2}}\right|_{d=d_{0}}-c_{0} / d_{0}^{2},
$$

这里 $d_{0}$ 是平衡键长, $c_{0}$ 是径向力常数. 由此便可求得 $U_{1}--21.0 \mathrm{eV}, U_{2}-57.4 \mathrm{eV}$.

Chadi 通过详细求解能带的方法求出的 $U_{2}$ 为 $44.8 \mathrm{eV}$, 根据他的估计, 这比真实值小约 $20 \%$ ，这意味着本文的 $U_{2}$ 比真实值大 $10 \%$ ，这说明对于振动问题，成键轨道近似与详细解能 带的方法的可恝性可以相比较.

\section{三、计萁结果与讨论}

GaAs（110）表面原胞如图 1, 二维布里洲区如图 2. 我们采用 Chadi ${ }^{[2]}$ 给出的表面结 构,在忽略杂化轨道指向与键方向不一致对总能量的影响的条件下,对 15 层原子作计算,得到

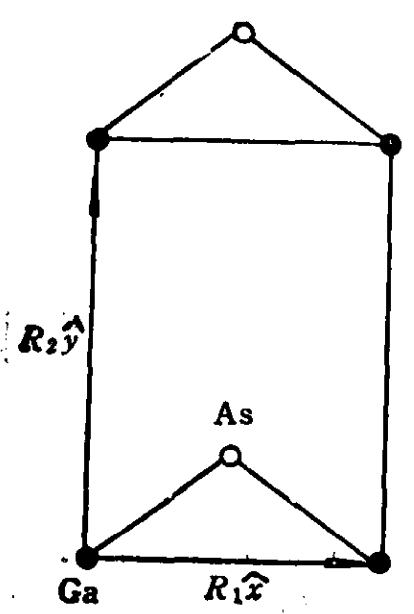

图 1 GaAs (110) 表面原胞

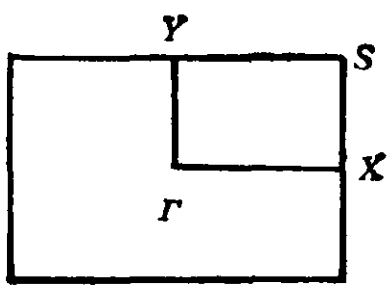

图 2 二维布里消区 


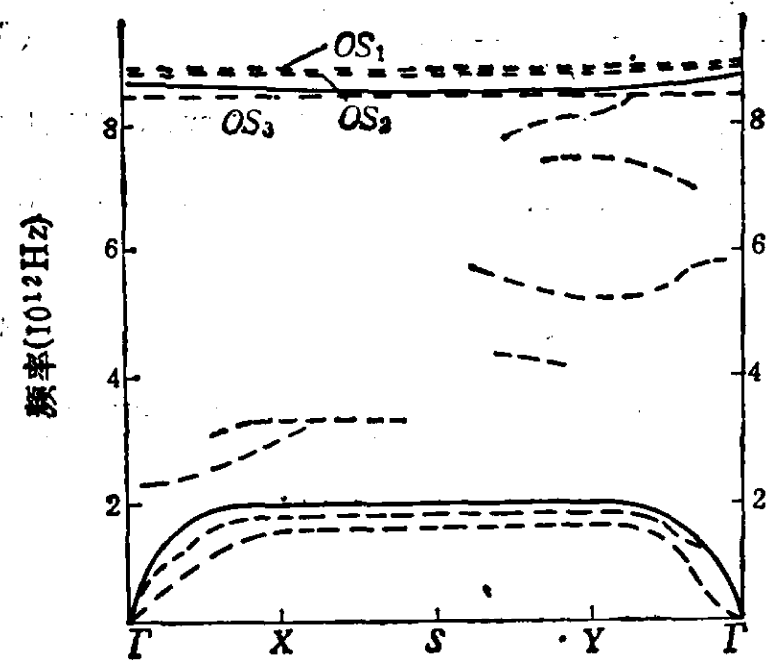

图 3 声子色散曲线

因中两实线给出了体带投的范国，应线表示表面楼

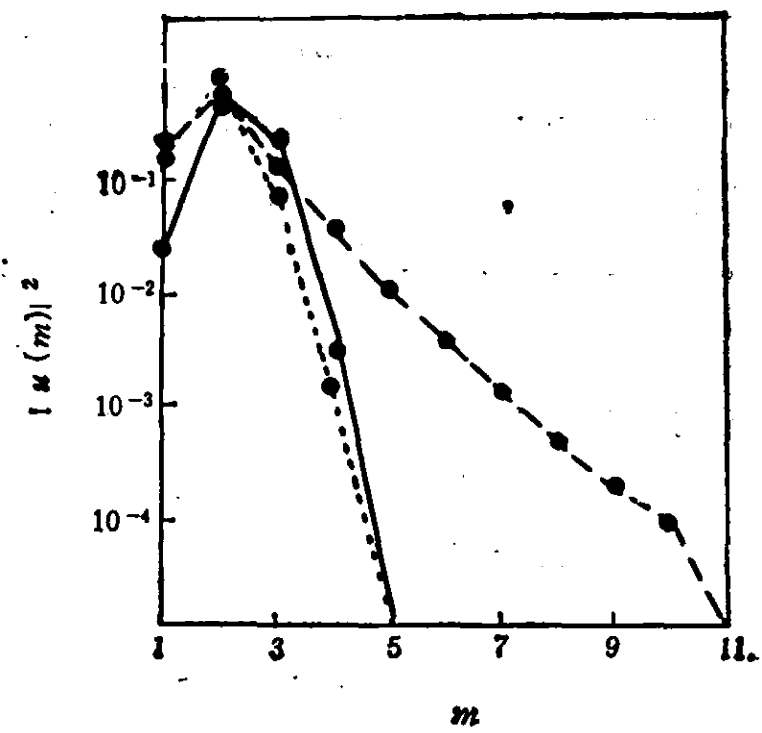

图 4 部分表面模向体内的衰减情况

$m$ 是原子层指标， $m=1$ 代表表面层。 $\mathrm{OS}_{1}, \cdots-\mathrm{OS}_{2}, \ldots \ldots \ldots \mathrm{S}_{3}$

的声子色散如图 3. 图 3 的色散曲线有两点是值得注意的:

（1）图中出现了高于体纵光学声子的表面模 $O S_{1}$ 及 $O S_{2}$ ，它们向体内的衰减见图 4. 由 于驰豫使得表面相互作用减弱, 通常认为清洁表面的表面声子能量低于 $L O$ 声子, 实际所测 的金属及离子性晶体表面确是如此.但是 $\mathrm{Mele}^{[3]}$ 等得到的 $\mathrm{Si}(100) 2 \times 1$ 表面振动也出现了 与本文相似的高能表面模，这是因为参与这种表面模振动的相邻原子较强烈地拉伸它们之间 所成的键造成了高振动频率.这种相似性可能不是偶然的,它导致了这种设想: 存在这种表面 模可能是半导体表面振动的特点之一, 这一点值得进一步研究. 由于本文所用理论本质上与 Mele 等所用的相同, 实验上对它们存在的确定将对这种振动理论的方法提供很好的检验。

(2) 接近体模声子顶端的表面模 $O S_{3}$ 是值得一提的, Ruppin ${ }^{(6)}$ 等利用黄昆方程在讨论 极性晶体时指出总存在能量在 $\omega_{\text {LO }}$ 与 $\omega_{\text {To }}$ 之间的表面模,最近在 GaP 表面的 Raman 散射的 测定证实了他的理论 ${ }^{[\eta]}$. 尽管连续介质的极化理论和分立晶格的紧束㭪方法似乎是两种完全 不同的理论形成,但其物理实质并无本质的不同,在这里也得到相似位置 $O S_{3}$ 表面模. 我们认 为 $O S_{3}$ 的出现仍可说是对本文方法的一个支持.

应该指出, $\mathrm{OS}_{3}$ 在能量上完全浸没在体光学声子的投影带中，因而不能按通常的方法在体 模投影带隙中找出,它完全是根据表面模的含义按照其本征矢的衰减情况确定出的. 这也是 本文不画出体投影带细节而只给出体带范围和表面模出现的位置的原因之一。

除了上面的结果，作者还用十一层及七层原子近似算了重构表面沿 $\Gamma X ， X S$ 对称线的声 子,结果表明即使用七层近似,表面模的位置都没有什么变化. 查看它们的本征矢很容易理解 这一点,因为它们向体内衰减都是非常快的,这就是说,在不考虑长程作用的情况下,只要考虑 少数几层原子便能使有很强的局域性的表面声子的计算结果达到相当的精度。虽然这会给缓 变的表面模造成一些误差,甚至无法辩认，对体模误差更可观. 但它可大大减少计算量，擗免 了表面问题的一大困难。而且，体模产生的不精确性无伤大雅,因为求体模的理论是较成熟的。 
此外, 我们还用 15 层平板近似计算了理想表面 $\Gamma X, X S$ 线上的声子色散, 发现它与重构表面 的声子色散有重大差别. $O S_{1}$ 及 $O S_{2}$ 不再出现, $O S_{3}$ 只在 $\Gamma$ 点附近存在. 其它表面模在能量 上也有较大变化. 这说明表面驰豫对表面声子起着决定性的影响.

\section{四、结 束 语}

用成键轨道近似计算能带总能量, 概念简单,计算量小、容易改进。它将力常数与电子结 构相联系, 既能预言声子谱, 又能很自然地给出由体内到表面力常数的变化. 且不存在从头计 算所遇到的难以克服的计算量. 因此, 对研究不同共价半导体的各种各样的重构表面的声子 谱是简单而有效的方法. 但由于只考虑了最近邻作用, 对描述与长程作用有关的声子显然是 不理想的. 此外,如何修正占据态与非占据态之间的耦合对能带能罯的影响还需要进一步的 考虑。

\section{宩文 堿}

[ 1 ] Soke1, R. C., Bull. Am. Phys. Soc., 21(1976), 1315.

[2] Chadi, D. J., Phys. Rev.. B19(1979), 2074; Phys. Rev. Lett. 52(1984), 1911.

[3] Weber, W. and Varma, C. M., Phys. Rev, B19(1979), 6142.

[1] Harrison, W., Electronic Structure and Propertics of Solids, Freeman, San Francieco, 1980.

[ 5 ] Mele, E. J., et al., J. Vac. Sci. Technol, B3(1985), 1068.

[6] Ruppin, R. and Englman, R. Rep. Prog. Phys., 33(1970), 149.

[7] Haysshi, S. and Ruppin, R., J. Phys, C18(1985), 2583. 